Methods: Pregnant SLE patients (diagnosis made according to ACR 1997 criteria) were included in the present study, conducted in the context of a joint rheumatology/gynecology multi-disciplinary team. For each patient we collected demographic information, medical history, treatments, disease activity (SLEDAI-2K) chronic damage (SLICC damage index), clinical and laboratory data, including serum complement level and autoantibodies. Pregnancy outcomes were reported longitudinally as well as disease relapses occurring during pregnancy and puerperium. Flares were defined as new onset or worsening disease-related manifestation in any organ/system.

Results: Since 2008, 70 consecutive pregnancies occurred in 50 SLE patients [(median age at diagnosis 25 years (IQR 12.2), median age at first pregnancy 33 years (IQR 7), median disease duration 72 months (IQR 120)]. As controls, we evaluated 100 consecutive pregnancies in 100 women without autoimmune diseases [(median age 31 years (IQR 9)]. Table 1 reports the obstetric, fetal and neonatal outcomes of SLE patients compared to control group. A positive outcome in terms of live born infants was experienced in $88.6 \%$ of SLE pregnancies and in $88 \%$ of control group $(p=N S)$. There were no statistically significant differences in any of the pregnancy outcomes evaluated; however, the percentage of small for gestational ages (SGA) was significantly higher in SLE group (22.8\% versus $11.0 \% \mathrm{P}=0.003)$. A statistical association was found between SGA and positivity for anti-dsDNA, anti-SSA ed anti-SSB $(p=0.0001, p=0.01, p=0.04$ respectively). Miscarriage was significantly associated with disease-related serologic abnormalities [anti-dsDNA $(p=0.0001)$, low $C 3(p=0.0001)$ and low $C 4(p=0.006)$ ] and past smoking habitus $(\mathrm{p}=0.0001)$; preterm birth was associated with anti-dsDNA, anti-CL and anti-B2GPI ( $p=0.001, p=0.0005, p=0.01$ respectively). A disease flare was reported in 28 pregnancies $(40 \%)$ and in 31 puerperium $(44.3 \%)$. Figure 1 reports SLE relapses divided according to organ involvement. Flare during pregnancy was associated with positivity for anti-SSA $(p=0.001)$, anti-SSB $(p=0.01)$ and $a-C L(p=0.006)$, while puerperium relapses were associated with previous renal involvement $(p=0.0005)$, flare during pregnancy $(p=0.01)$ and chronic damage $(p=0.0001)$.

Table 1. Pregnancy outcomes in $\mathbf{5 0}$ SLE and 100 controls.

\begin{tabular}{lccc}
\hline & $\begin{array}{c}\text { LES } \\
\text { (Pregnancies N=70) }\end{array}$ & $\begin{array}{c}\text { Controls } \\
\text { (Pregnancies N=100) }\end{array}$ & P \\
\hline OBSTETRIC OUTCOME & & & \\
Preterm birth N/\% & $18 / 25.7$ & $19 / 19$ & NS \\
Gestational hypertension N/\% & $5 / 7.1$ & $3 / 3$ & NS \\
Gestational diabetes N/\% & $5 / 7.1$ & $5 / 5$ & NS \\
Pre-eclampsia N/\% & $2 / 2.9$ & $1 / 1$ & NS \\
FETAL OUTCOME & $8 / 11.4$ & $12 / 12$ & NS \\
Miscarriages N/\% & $4 / 6.4$ & - & - \\
PR interval elongation N/\% & $3 / 5$ & $1 / 1$ & NS \\
IUGR N/\% & $16 / 22.8$ & $11 / 11$ & $\mathbf{0 . 0 0 3}$ \\
NEONATAL OUTCOME & $2850-688$ & $3250-814$ & $\mathbf{0 . 0 0 3}$ \\
SGA< 10 centile N/\% & $8-1$ & $8-1$ & NS \\
Weight at birth median-I.Q.R. & $9-1$ & $10-1$ & NS \\
Apgar 1' median-I.Q.R. & & &
\end{tabular}

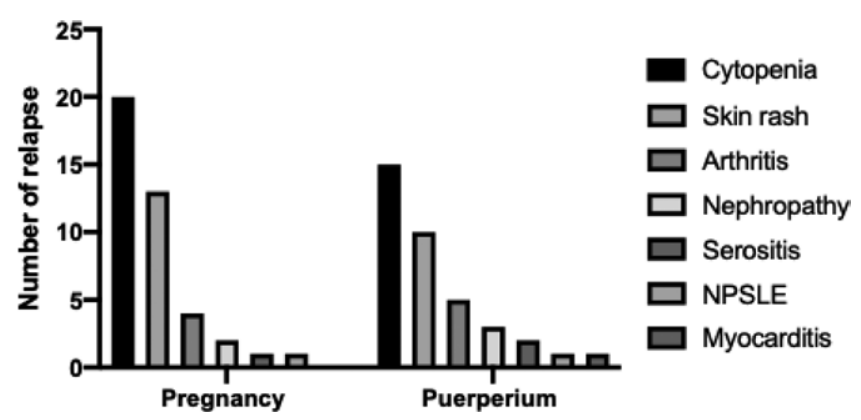

Figure 1. Disease flares during and after 70 SLE pregnancies divided according to organ involvement.

Conclusion: The present study confirms the role of pre-gestational counseling and a multi-disciplinary approach in the outcome of SLE pregnancies. Moreover, the high prevalence of disease relapse even more justifies the need for a combined rheumatology/gynecology multi-disciplinary approach.

Disclosure of Interests: Carmelo Pirone: None declared, Fulvia Ceccarelli: None declared, Aikaterini Selntigia: None declared, Carlo Perricone: None declared, Simona Truglia: None declared, viviana antonella pacucci: None declared, Francesca Romana Spinelli Grant/research support from: Pfizer, Speakers bureau: Lilly, BMS, Celgene, cristiano alessandri Grant/research support from: Pfizer,
Guido Valesini: None declared, Giuseppina Perrone: None declared, fabrizio conti Speakers bureau: BMS, Lilly, Abbvie, Pfizer, Sanofi

DOI: 10.1136/annrheumdis-2020-eular.3106

\begin{tabular}{|l|l}
\hline SAT0229 & PROTEOMIC ANALYSIS REVEALS ASSOCIATION \\
BETWEEN IMMUNE-METABOLIC BIOMARKERS AND \\
CLINICAL SYMPTOMS IN SICCA PATIENTS
\end{tabular}

V. Pucino $^{1,2}$, J. D. Turner ${ }^{1}$, F. Kollert ${ }^{3}$, S. Rauz ${ }^{1,4}$, A. Richard ${ }^{5}$, J. Higham ${ }^{5}$, A. Poveda-Gallego ${ }^{1,5}$, S. J. Bowman ${ }^{1,2}$, F. Barone ${ }^{1,2}$, B. Fisher ${ }^{1,2} .^{1}$ Institute of Inflammation and Ageing, College of Medical and Dental Sciences, University of Birmingham, UK, Birmingham, United Kingdom; ${ }^{2}$ National Institute for Health Research (NIHR) Birmingham Biomedical Research Centre and Department of Rheumatology, University Hospitals Birmingham NHS Foundation Trust, Birmingham, UK, Birmingham, United Kingdom; ${ }^{3}$ Department of Rheumatology, Immunology, and Allergology, Inselspital, University Hospital Bern, Bern, Switzerland, Bern, Switzerland; ${ }^{4}$ Academic Unit of Ophthalmology, Birmingham and Midland Eye Centre, Birmingham, UK, Birmingham, United Kingdom; ${ }^{5}$ Department of Oral Medicine, Birmingham Dental Hospital, Birmingham, UK, Birmingham, United Kingdom

Background: Sjögren's syndrome (SS) is a systemic autoimmune disease whose main characteristic is involvement of the exocrine glandular system. Thus, its most common clinical manifestation is eye and mouth dryness which, alongside fatigue and pain, contributes to poor health-related quality of life (HRQoL). A growing body of evidence recognises the adipose tissue as an active endocrine organ secreting bioactive mediators involved in metabolic and inflammatory disorders. A relationship between obesity and symptoms in SS has not yet been elucidated.

Objectives: To explore potential associations between obesity-related immune-metabolic biomarkers and clinical symptoms in SS and sicca patients.

Methods: Proteomics analysis of 184 cardio-immuno-metabolic proteins was assessed on sera from 53 SS (50 females (F), 3 males (M); mean age 54 years) and 60 sicca ( 56 F, 4M; mean age 57 years) patients. Participants were enrolled in the Birmingham Optimising Assessment in Sjögren`s Syndrome (OASIS) cohort and examinations included the EULAR SS Patient Reported Index (ESSPRI), Schirmer's test, unstimulated whole saliva, minor labial salivary gland biopsy, EuroQoL-5 dimension (EQ-5D) tool, immunological parameters, body max index (BMI) and $\mathrm{Hb} 1$ Ac. Participants with SS fulfilled 2016 ACR/EULAR criteria. Non-SS sicca patients were anti-Ro antibody negative, displayed objective oral and/or ocular dryness, and did not have a physician diagnosis for SS. Linear regression analysis and univariate models were performed to identify the key predictors of symptoms. Results: HRQoL as measured as EQ-5D utility values, symptoms as assessed by ESSPRI, and BMI did not differ between the SS and sicca group. However, strong correlations between BMI (or fat or fat-free mass) and EQ-5D and ESS$\mathrm{PRI}$ scores were found in the sicca but not in the SS group. Among several proteins investigated, ADM, TNFRSF13B, FGF23, IL10RB, CD5, CD40, IL1RA, TNRSFN9, TNFRSF10A, TNFRSF11A, TRAILR2, GAL9, SPON2, ACE2, LEP, CD4, IL12B, SLAMF1, PD-L1 positively correlated with symptoms in the sicca group $(R \geq 0.3$; Holm's adjusted $P \leq 0.05)$. Adrenomedullin $(A D M)$ showed the strongest correlation with ESSPRI $(R=0.56$; $P<0.0001)$; age $(R=0.45 ; P=$ $0.0003)$; $B M I(R=0.43, P=0.0008)$; Ocular Surface Disease Index $(R=0.32$, $P=0.03)$; EQ-5D utility value $(R=-0.45, P=0.001)$ and VAS patient $(R=-0.41$, $P=0.008)$. There was no association between ADM and gender, Schirmer's test, disease and symptom duration $(P>0.05)$. ADM was independently associated with ESSPRI scores in non-SS sicca patients when corrected for BMI, age, $\mathrm{HbA1c}$, depression and anxiety scores. None of the above mentioned proteins correlated with clinical symptoms in the SS group.

Conclusion: The study suggests that obesity-related immune-metabolic factors may play a role in regulating the symptoms in non-SS sicca patients. ADM appears to be a strong independent predictor of symptoms in these patients but not in SS.

Disclosure of Interests: Valentina Pucino: None declared, Jason D. Turner: None declared, Florian Kollert Employee of: Novartis, Saaeha Rauz: None declared, Andrea Richard: None declared, Jon Higham: None declared, Ana Poveda-Gallego: None declared, Simon J. Bowman Consultant of: Astrazeneca, Biogen, BMS, Celgene, Medimmune, MTPharma, Novartis, Ono, UCB, xtlbio, Glapagos, Speakers bureau: Novartis, Francesca Barone: None declared, Benjamin Fisher: None declared

DOI: 10.1136/annrheumdis-2020-eular.2900

\section{SAT0230 MACROPHAGE ACTIVATION SYNDROME IN SLE AND SYSTEMIC ONSET JIA: SIMILAR OR DISSIMILAR}

N. R ${ }^{1}$, A. Jain ${ }^{1}$, H. Muhammed ${ }^{1}$, A. Aggarwal ${ }^{1}$, V. Agarwal ${ }^{1}$, L. Gupta ${ }^{1}$, D. Misra ${ }^{1}$, A. Lawrence ${ }^{1}$, R. Misra ${ }^{1} .{ }^{1}$ SGPGI, Clinical Immunology and Rheumatology, Lucknow, India 